\title{
Double Purse String Closure Technique for Minimizing Post-Operative Widening of Periareolar Augmentation Mastopexy: A Comparative Study
}

\author{
NAHED S. BOUGHDADY, M.D. \\ The Department of Plastic and Reconstructive Surgery, Faculty of Medicine, Ain Shams University, Cairo, Egypt
}

\begin{abstract}
Objectives: For management of mild degree breast ptosis periareolar mastopexy had been commonly used. Since Benelli 1990 had confined the scar to the areola in his round-block technique, several authors has adopted this technique. Whether mastopexy had been done with or without augmentation, the problems of breast shape, areolar irregularities, and stretch of the areola had been annoying to both patients and surgeons. These problems also represent a major challenge to this technique. In this paper purse string closure was used in two consecutive different layers one in dermal de-epithelized layer and another in the deep dermis of the skin. The aim is to minimize widening of scars of peri-areolar incision and improve its long term appearance.
\end{abstract}

Patients and Methods: 50 cases underwent mastopexy with breast augmentation. Cases were divided into two groups: Group A: Underwent closure by single purse string layer and Group B: Two consecutive purse string closure. The second concentric row was applied to the deep dermal plane of the normal non de-epthelialized skin similar to the classical purse string closure described previously. Both subjective and objective evaluation were done. Surgery was done by single surgeon and two surgeons not involved in research evaluated the post-operative photos. Follow-up was done at 3,6 month and at 1 year post-operative.

Results: Group B patients showed superior results as regarding breast shape, contour, projection, superior fullness, periareolar scar shape, regularity, areola width and position. These results were followed-up for one year.

Conclusion: Decreasing the tension of the periareolar suture by applying double purse string technique in two different planes improved long term results of periareolar scar in order to avoid widening, irregularities and hypertrophic scarring.

Key Words: Breast ptosis - Periareolar - Mastopexy - Augmentation.

\section{INTRODUCTION}

Several factors as aging, infirmity, gravity and loss of weight results in ptotic breast which leads to loss of its attractiveness. The female breast is one of the most important feminine characters. In surgeons considerenig treatment of ptosis, its very important to balance shape, volume, and scars with a low recurrence rate. Over decades of breast mastopexy and augmentation, surgeons had strug- gled with long-term results and reccurence of ptosis. Regnault had classified breast ptosis according to the position of the nipple relative to the inframammary fold while the patient is in standing position [1].

A breast condition in which the gland is in inferior position to the inframammary crease line and the nipple is above it is called pseudo ptosis. When the nipple is at or up to $1 \mathrm{~cm}$ below the infra mammary crease this is Grade I ptosis. While the nipple at a level 1 to $3 \mathrm{~cm}$ below the crease this is Grade II. In Grade III ptosis the nipple is more than $3 \mathrm{~cm}$ below the crease or at the inferior pole of the breast. The inferior pole Nipple-Areolar Complex (NAC) (Grade III) is designated as an end point of ptosis, although the same anatomic configuration may occur in a tubular breast deformity with a high inframammary fold and lower pole parenchymal hypotrophy, as mentioned by Brink [1,2].

Mastopexy augmentation is gaining a lot of popularity nowadays. It doesn't depend on the position of the mammary implant whether subglandular or submuscular alone to adequately correct sagging skin. Also depending on a surgical upper relocation of the pre-existing tissues to treat ptosis alone is not always enough. Both of these factors are not sufficient to restore skin tightness, shape, and volume (especially the upper pole fullness) of a young breast. For correction of mild to moderate degree ptosis periareolar mastopexy techniques are most often recommended [3-5].

Peri areolar incisions yield the least scarring which encouraged several authors to perform augmentation and mastopexy in one procedure. Some authors stated that it is a safe and effective procedure while others claimed that this causes a significant degree of risk and increase complications of the procedure with increased degree of recurrence of ptosis in those patients with combined procedure [6-12]. 
In peri-areolar mastopexy there is two forces influencing the operation, in order to confine the scar to the areola, and get the ptotic breast well supported for long period without recurrence. These forces include degree of breast ptosis, implant position shape and size and other factors that create a lot of tension on the scar. Benelli, Bartels and Erol stressed on the value of glandular reshaping, suspension and used the purse-string blocking suture to minimize the scars. Mixed mesh has been used by Goes to provide long-term support of the gland [3-16].

This study suggests a modification of the technique of suturing to eliminate these complications. The aim of this work is to evaluate the effectiveness of a new double purse string technique used in order to reshape the breast, maintain projection, adjust the size of the areola, avoid tension on the areola and prevent uguly scarring. Also other complication such as irregularities, hypertrophic scar, areolar spreading stitch marks can be minimized.

\section{PATIENTS AND METHODS}

50 patients underwent mastopexy for mild to moderate degree ptosis, when measuring nipple areola complex position from mid clavicular point (21-25cm). Study was done in Plastic and Reconstructive Surgery Department of Ain Sams University in the period from 2013-2014. Ages ranged from 28-35. All cases had undergone augmentation mastopexy using silicone implants sized ranging from 200-300. 44 cases were primary and 6 cases were secondary revision surgery of widened areolar scars. All patients underwent periareolar augmentation mastopexy, de-epithelialized dermal sleeve and glandular reshaping.

Inclusion criteria included middle aged patients, moderate degree ptosis $3-5 \mathrm{~cm}$, with no history of smoking, DM, last breast feeding 2 years ago. BMI ranges from 23-32 and excluding patients with massive weight loss. The nipple is measured 21$25 \mathrm{~cm}$ from the mid clavicular line in passing by nipple areolar complex down to inframammary fold.

\section{Marking of surgery:}

Pre-operatively skin markings was done according to Gonzaliz 2012 with some modification. The markings are done with patient standing. The midclavicular vertical line was marked first, passing from the middle of the clavicle to the center of the areola bilaterally (line drawn from midclavicle to NAC). The highest point of the circumareolar marking (Point $\mathrm{H}$ ) was drawn over this midclavicular line with a ruler. Gonzaliz determined the location of Point $\mathrm{H}$, a caudal distance was measured from the highest point on the clavicle, corresponding to the patient's height in centimeters divided by 10 . The lowest point of the areola was then marked (Point L). In this study Point $\mathrm{H}$ was located at 19$21 \mathrm{~cm}$ on the line drawn from the midclavicle to the NAC and this measurement difference was guided by the degree of ptosis and size of implant. Points $\mathrm{H}$ and $\mathrm{L}$ were connected through oval drawing which determine the de-epithelized area. The new areola size is marked in most cases, to range from 4 or $4.2 \mathrm{~cm}$. This (HL) shape is first oval, to be closed around the new areola as rounded Fig. (1).

Mastopexy was done according to Periareolar Augmentation Mastopexy (PAM) technique Gonzaliz 2012 and the implant size ranged from 200-300 [17]. This is considered as moderate size, so we can depend on mastoxpey alone for lifting not on prosthesis size and also to avoid future ptosis. Projection of the implant ranged from 4.5 to $5.9 \mathrm{~cm}$. The small size of silicone was safer to prevent problems of big prosthesis and its applied forces on the areola while the high projection improved shape and upper pole fullness. All implants introduced in the subglandular plane were smooth and round and this plane was chosen to avoid the double bubble appearance. The marked point of the middle of upper pole of the areola is sutured to the upper skin by a buried stitch with 2-0 polydiaxonone in all cases. This was done in order to prevent rotation of the areola.

Patients were divided into two groups whereas periareolar closure technique was done in all cases. Group A included 25 cases in which classic closure was done through a two layer approach. Closure of the skin lining purse-string suture (which will determine the new areola diameter) using a 2-0 polydiaxonone (PDS, Ethicon). Keep the suture line in the deep intradermal position. Tie this suture around a $4-\mathrm{cm}$ tube so that the resulting NAC shape (with a $4-4.2 \mathrm{~cm}$ diameter) is slightly convex. Suture the areola to the skin lining using a non-interrupted 4-0 Monocryl (Ethicon) intradermal suture. This suture should be distributed between skin $4 \mathrm{~mm}$ to $1 \mathrm{~mm}$ of NAC to minimize periareolar wrinkle formation and to achieve a symmetrical rounded final appearance.

In Group B (25 cases) purse string closure was performed in two concentric rows. The first concentric suture was applied circumferentially throughout the middle of the de-epithelialized skin passing in the deep dermal layer.

The second concentric row was applied to the deep dermal plane of the normal non de-epthelialized skin similar to the classical purse string closure described previously Fig. (2). 

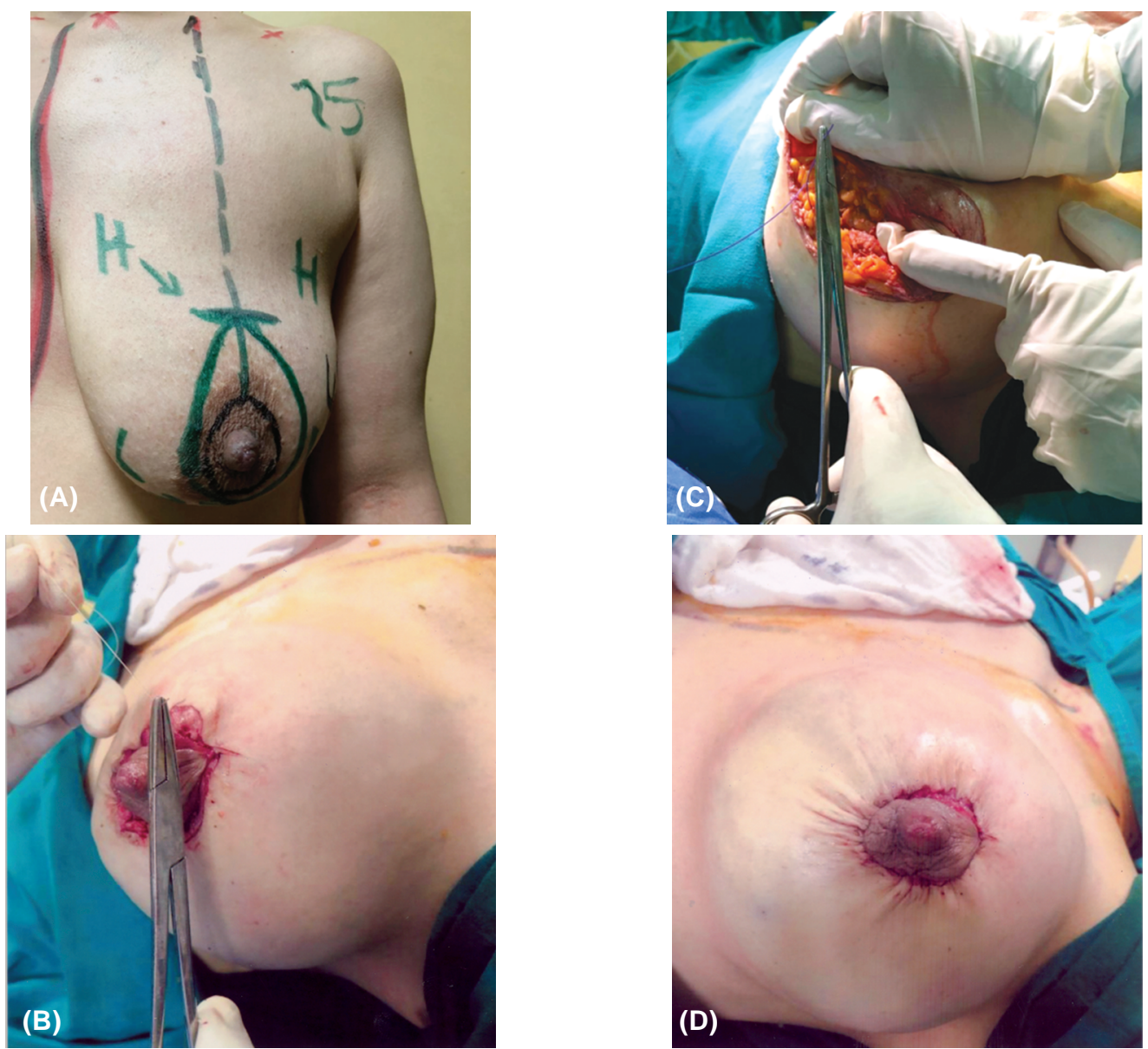

Fig. (1): (A-D): A: Surgical landmarks of peri-areolar augmentation mastopexy, B: Plication of bilateral pillars, C: First purse string while, D: After second purse string.
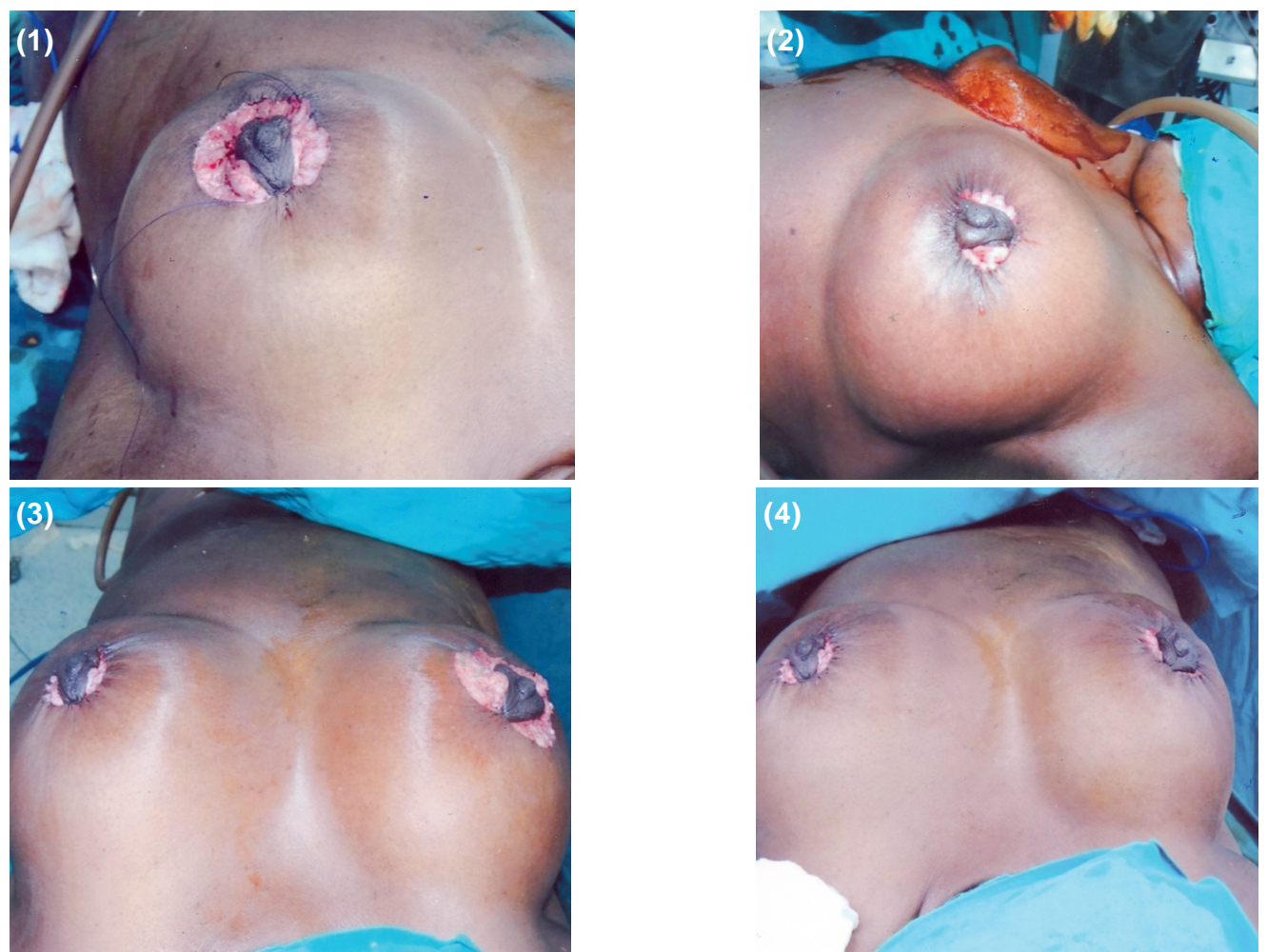

Fig. (2): Picture (1); Left breast with the first concentric intra dermal purse string, Picture (2); Same breast after second concentric row, Picture (3); Left breast after two concentric rows while Rt breast after one row with big difference in circumference between both areola, Picture (4); Breast bilaterally after two concentric rows. 
Table (1): Patients' data.

\begin{tabular}{cccc}
\hline & Age & Primary & Secondary \\
\hline Group A & $28-32$ & 21 & 2 \\
Group B & $29-35$ & 23 & 4 \\
\hline
\end{tabular}

Third layer of closure; non-interrupted 4-0 Monocryl (Ethicon) intradermal suture. This suture should be distributed between external skin $4 \mathrm{~mm}$ to $1 \mathrm{~mm}$ of NAC to minimize periareolar wrinkle formation and to achieve a symmetrical rounded final appearance.

Subjective evaluation was done by assessment of patient satisfaction from the final result, breast position and toning and the circum-areolar scar in correlation to the early post-operative one. Patient satisfaction was ranked on scale of 0-3 with 0 being dissatisfaction and 3 being highly satisfied ( 0 degree means that scar shows signs as hypertrophy, redness, irregularities and widening. 1 degree means no irregularities but widening and redness. 2 degree means no irregularities or hypertrophy and mild widening. 3 degrees means that breast is firm lifted with good shape and scar is totally concise, small with no widening at all or any other changes). The perception of scar appearance is measured through the Vancouver scar scale. Objective evaluation was done by assessment of the outcome of surgery 3,6 and 12 months postoperatively by comparing the pre-and postoperative photos as regards the final aesthetic outcome by two plastic surgeons not involved in the treatment and one nurse and they gave scores for the cases. Their evaluation was recorded as percentage on grading scale: $<25 \%$ : Poor, $25-50 \%$ : Moderate, 51-75\%: Good and 76-100\%: Excellent result.

\section{RESULTS}

Follow up of the patient was done for one year, post-operative photos were done at 3,6 months and 1 year. The overall complication rate in Group A was $20 \%(n=5)$ and in Group B was $12 \%(n=3)$. The complications encountered in the study was sloughing of the edges in 4 cases mild infection responded to treatment by antibiotics and seroma in 1 case (Table 2).

Subjective evaluation revealed overall high grade of satisfaction in 12 cases in Group A and 20 cases in Group B (Table 3).

Objective evaluation of the final aesthetic outcome in Group A was excellent in 5 cases, good in 13 cases. While in Group B it was excellent in 15 cases and good in 8 cases. Vancouver scar scale was used to assess scar quality. In Group A scar scale was 3 while in group $B=7$ as scars were narrower, softer and of of better quality with less irregularities. Follow-up was also done at 6 months and one year (Table 4).

Table (2): Complications in both groups.

\begin{tabular}{lcc}
\hline & Group A & Group B \\
\hline Superficial sloughing of edges & 3 & 2 \\
Infection & 1 & 1 \\
Hematoma & - & - \\
Seroma & 1 & - \\
\hline
\end{tabular}

Table (3): Results of subjective evaluation.

\begin{tabular}{ccccc}
\hline & $\begin{array}{c}\text { High } \\
\text { satisfaction } \\
(3)\end{array}$ & $\begin{array}{c}\text { Good } \\
\text { satisfaction } \\
(2)\end{array}$ & $\begin{array}{c}\text { Low } \\
\text { satisfaction } \\
(1)\end{array}$ & $\begin{array}{c}\text { Dis- } \\
\text { satisfaction } \\
(0)\end{array}$ \\
\hline Group B & 20 & 3 & 1 & 0 \\
Group A & 12 & 5 & 2 & 6 \\
\hline
\end{tabular}

Table (4): Results of objective evaluation.

\begin{tabular}{lcccc}
\hline & Excellent & Good & Fair & Bad \\
\hline Group A & 5 & 13 & 6 & 1 \\
Group B & 15 & 8 & 2 & 0 \\
\hline
\end{tabular}
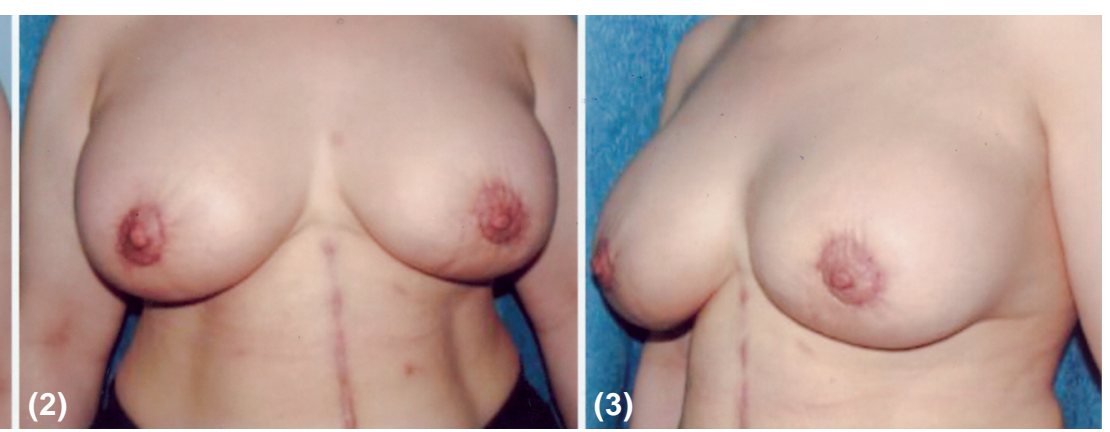

Fig. (3): Case of Group A pre-operative Photo (1), and Pictures post-operative $(2,3)$. 

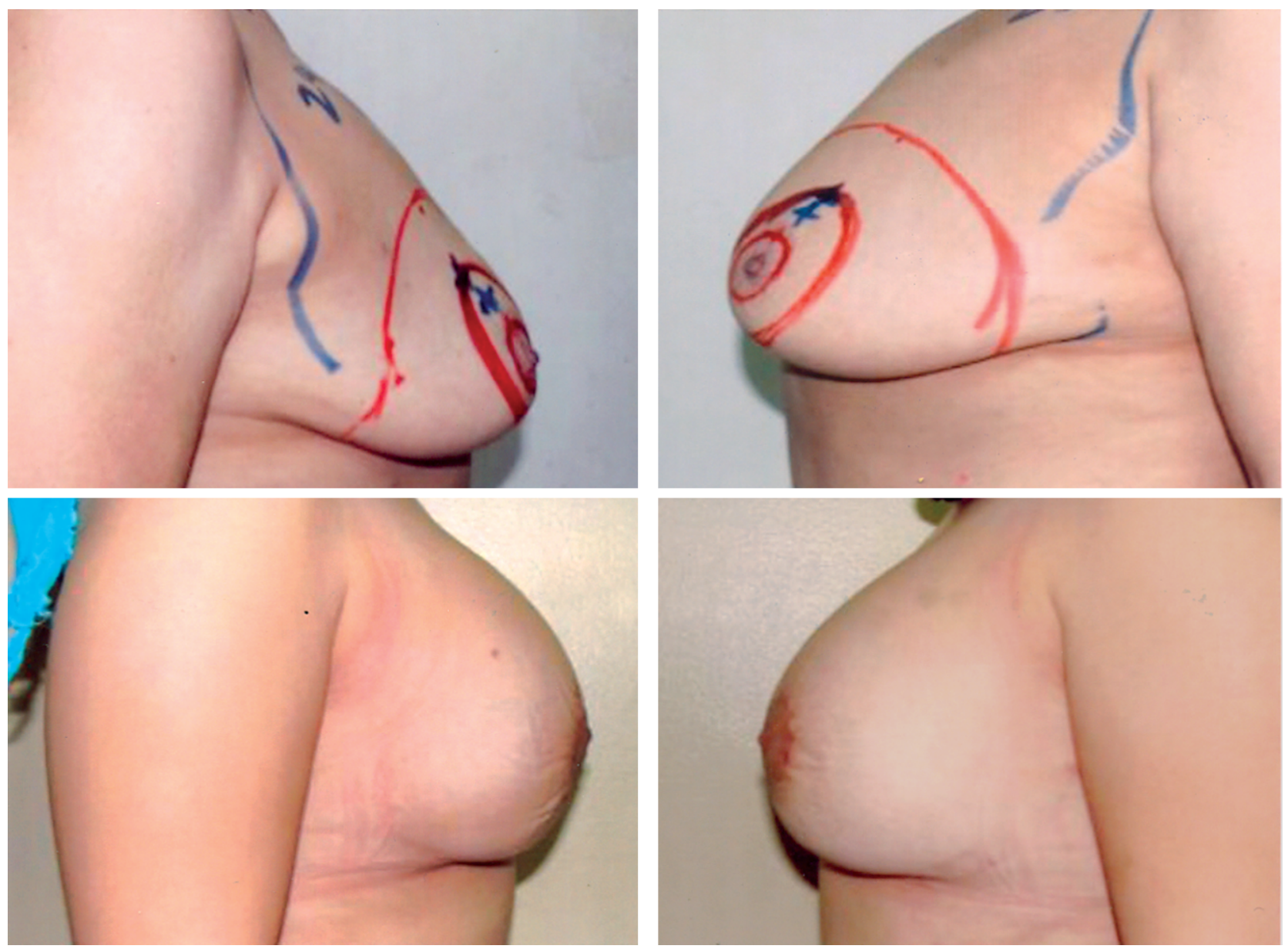

Fig. (4): Case of Group B, 1 and 2 pre-operative Rt, Lt dead lateral photos and post-operative 3, 4 Rt, Lt dead lateral photos.

\section{DISCUSSION}

Surgeons performing mastopexy augmentation need to provide good support to breast gland to overcome different forces affecting the procedure as aging, gravity and breast feeding all through the least sacr possible. Stable circular shape with least complications and long term results are their main concerns. For mastopexy there are three skin closure patterns; the T pattern, the vertical and the circular periareolar pattern.

For the vertical method, Hall-Findlay had used the superior and medial pedicle to advance the principles of breast shaping. Several authors tried to minimize the size of the scar as Hammond who combined a mix of peri-areolar and short-vertical scar principles. Compared with the other methods strong centrifugal forces affect the minimal-scar concentric method of mastopexy. This becomes more evident in applying this method to more complicated and severe cases of ptosis $[\mathbf{1 8 , 1 9 ]}$.

Bass introduced the deep double-layered closure in order to reduce the tension on the areolar suture line when used in cases of minimal or moderate hypertrophy or ptosis. It also minimizes postoperative widening of the constructed areola and allows immediate skin contouring with minimal skin scar. Similar technique as doughnut mastopexy also tried to reduce the size of the areola in some selected cases [20,21].

Since then several authors adopted the concentric mastopexy procedure as an application for mild glandular ptosis, and areola asymmetry, as well as in cases of treatment of tuberous breast. Simulataneous augmentation mastopexy is recorded to be safe and effective, it is recommended for patients who have unadequate breast volume but wish for more upper pole fullness, firmness and projection but carry a risk of sertain undesired sequel $[6,7]$.

The principal advantage of periareolar purse string closure is confining the scar to the areola, the problem is mainly widening which was partly minimized by purse string suture which gathers the excess skin in order to minimize this widening and decrease the centrifugal tension for better healing [22]. 
Goes usied polyglactine-polyester mesh to perform the periareolar mastopexy or reduction technique in order to obtain a conical shape of the breast. This provided him with stable results instead of depending on skin envelope alone which does not prevent early recurrence or areola enlargement. several authors also used permenat materials. Other authors as Chapman used Gortex suture in periareolar closure $[\mathbf{1 6 , 2 3 , 2 4 ]}$.

In this study only absorbable sutures were used in all layers of closure only polydiaxonone. This was done in both groups although in Group A the closure was in two layers but in Group B closure was done in three layers. This was done as some patients feel annoyed by the sensation of non absorbable sutures.

Hickman also used periareolar mastopexy in cases of more moderate to severe cases $4-9 \mathrm{cms}$ ptosis he adopted the technique of Goes double skin periareolar mastopexy without mesh and used purse string suture 2/0 nylon permenant suture. Several studies preferred the periareolar mastopexy is better used with an adjuvant implant in cases of upper pole emptiness. Through this approach they reduce the areolar diameter and simultaneously elevate nipple position [16,19-21,25].

Purse string technique is one of the widely used approaches in order to prevent bottoming out and preserving long term projection of periareolar breast lifting. Others as zigzag wavy line periareolar incison by Gryskiewicsz [26].

In our study we did augmentation mastopexy in all cases using periareolar approach. All cases had mild to moderate degree ptosis $3-5 \mathrm{~cm}$ we used moderate size implants $200-300 \mathrm{cc}$ high profile implant only rounded smooth. The periareolar approach was safe and effective in this degree of ptosis, implants were important for upper pole fullness also helped in projection of the elevated breast. The small size implant was safe as regarding vascularity and produced least tension on the scar.

Gonzaliz used circumareolar incision to lift NAC $3-4 \mathrm{~cm}$ and reduce excess skin, associated with augmentation with implants sizes ranged from 199 to $285 \mathrm{cc}$ submuscular position. He used his marking of the highest point of elevation guided by patients height to present $10 \%$ of patients height [17]. In this study same points were used except that the highest Point $\mathrm{H}$ of marking was done according to mid humeral point $(19-21 \mathrm{~cm})$ according to the line drawn midclavicular to (NAC). The implants used in this study were 200-300 sub glandular position.
Cases in this study were divided in two groups. Marking and technique of peri-areolar mastopexy followed Gonzaliz 2012 with previous mentioned modifications. Technique of closure differed between the two groups. All in Groub A we used a single purse string technique and double layed closure. Purse string suture has to be pulled adequately over ( $4 \mathrm{~cm}$ tube) before appling the knot. In second layer the areola is sutured to the external skin using a non-interrupted intradermal suture in order to distribute the excess skin with minimum irregularities.

In Group B we modified this purse string suture in order to avoid its complications and get the highest benefit of it. We closed the skin in three rows instead of two first suture, was applied to deep dermal plane of the de epithelized skin in order to minimize the tension on the more superficial intra dermal plane of width and tension applied by suturing skin to areola. The second row is the regular purse string using absorbable polydiaxonone and the third row as in Group A. This row is also very important in order to use small bites to minimize the skin festooning of the pure string suture, also bury the knot and distribute the skin to areola in order of $4-1 \mathrm{~mm}$.

The sling suture of the upper pole of the areola to the H Point was very important in both groups as it prevents twisting of the areola and it was also buried in upper skin to prevent pointing of the stitch. Tha main advantage of this modification in Group B in addition to confinening the scar to the areola is the long term results regarding widening of the scar more projection of he areola and tidy appearance. Also to avoid severe tightening applied on the purse string suture to avoid recurrence widening. So instead of applying tension on one purse string it was distributed on both rows to get better long lasting effect and also avoid excessive festooning and delayed healing of single purse string.

Both groups were evaluated both subjective and objective. Subjective evaluation was measured through patient satisfaction which was ranked on scale of $0-3$ with 0 being dissatisfaction and 3 being highly satisfied. Group A showed 12 patients scale 3 and 6 patients scale 0 while Group B showed 20 patients scale 3 and 0 patients scale 0 .

Objective evaluation was done through evaluation of the pre and post-operative photos by two surgeons not involved in the procedure and one nurse. The final aesthetic outcome in Group A was excellent in 5 cases, good in 13 cases. 
While in Group B it was excellent in 15 cases and good in 8 cases. In Group B scars were narrower, softer and of better quality with less irregularities with scar scale 7 in comparison to scar scale 3 in Group A.

\section{Conclusion:}

Augmentation mastopexy can be done safely through periareolar scar. Decreasing the tension of the periareolar closure by applying double purse string technique in two different planes; one in the deepithelized dermis and the other on the non de epithelized skin not only improved the long term results but also improved areolar NAC projection and breast shape. The possibilities of widening, irregularities and hypertrophic scarring also decreased with increased patient satisfaction.

\section{REFERENCES}

1- Regnault P.: Breast ptosis: Definition and treatment. Clin. Plast. Surg., 3: 193-203, 1976.

2- Brink R.R.: Management of true ptosis of the breast. Plast. Reconstr. Surg., 91: 657-62, 1993.

3- Spear S.L., Boehmler J.H. $4^{\text {th }}$ and Clemens M.W.: Augmentation/mastopexy: A 3-year review of a single surgeon's practice. Plast. Reconstr. Surg., 118: 136S-47S, 2006.

4- Hoffman S.: Some thoughts on augmentation/mastopexy and medical malpractice. Plast. Reconstr. Surg., 113: 1892-3, 2004.

5- Nahai F., Fisher J., Maxwell P.G. and Mills D.C. II: Augmentation mastopexy: To stage or not. Aesthet. Surg. J., 27: 297-305, 2007.

6- Spear S.L. and Giese S.Y.: Simultaneous breast augmentation and mastopexy. Aesthet. Surg. J., 20: 155-63, 2000.

7- Spear S.L., Pelletiere C.V. and Menon N.: One-stage augmentation combined with mastopexy: Aesthetic results and patient satisfaction. Aesthetic Plast. Surg., 28: 25967, 2004.

8- Gallent I.M., Pons M.R. and Drever M.: Vertical scar mastopexy with an implant. Aesthetic Plast. Surg., 27: 406-10, 2003.

9- Karnes J., Morrison W., Salisbury M., Schaeferle M., Beckham P. and Ersek R.A.: Simultaneous breast augmentation and lift. Aesthetic Plast. Surg., 24: 148-54, 2000.

10- Stevens W.G., Stoker D.A., Freeman M.E., Quardt S.M., Hirsch E.M. and Cohen R.: Is one-stage breast augmen- tation with mastopexy safe and effective? A review of 186 primary cases. Aesthet. Surg. J., 26: 674-81, 2006.

11- Stevens W.G., Freeman E.M., Stoker D.A., Quardt S.M., Cohen R. and Hirsch E.M.: One-stage mastopexy with breast augmentation: A review of 321 patients. Plast. Reconstr. Surg., 120: 1674-9, 2007.

12- Tessone A., Millet E., Weissman O., et al.: Evading a surgical pitfall: Mastopexy-augmentation made simple. Aesthetic Plast., 2011.

13- Bartels R.J., Strickland D.M. and Douglas W.M.: A new mastopexy operation for mild to moderate ptosis. Plast. Reconstr. Surg., 57: 687, 1976.

14- Erol O.O. and Spira M.: A mastopexy technique for mild to moderate ptosis. Plast. Reconstr. Surg., 65: 603, 1980.

15- Benelli L.: A new periareolar mammaplasty: Round block technique. Aesth. Plast. Surg., 14: 99, 1990.

16- Sampaio-Goes J.C.: Periareolar mammaplasty with mixed mesh support: The double skin technique. Proceedings of the Eighth International Congress on Breast Diseases and Senology. Rio De Janeiro, Brazil, May 8e12, 1994.

17- Gonzalez R.M.D.: The PAM method-periareolar augmentation mastopexy: A personal approach to treat hypoplastic breat with Moderate Ptosis Aesthetic Journal, 32 (2): 17585, 2012.

18- Hall-Findlay E.J.: Pedicles in vertical breast reduction andmastopexy. Clin. Plast. Surg., 29: 379-91, 2002.

19- Hammond D.C., Alfonso D. and Khuthaila D.K.: Mastopexy using the short scar periareolar inferior pedicle reduction technique. Plast. Reconstr. Surg., 121: 1533-9, 2008.

20- Bass C.D.: Herniated areolar complex. Ann. Plast. Surg., 1 (4): 402, 1978.

21- Gruber R.P. and Jones H.W.: The donut mastopexy indications and complications. Plast. Reconstructive Surg., 65: 34, 1980.

22- Felicio Y.: Periareolar reduction mammoplasty. Plast. Reconstructive Surg., 88: 1789.

23- Hidalgo D.A. and Spector J.A.: Mastopexy. Plast. Reconstr. Surg., 132: 642e-656e, 2013.

24- Jade Chapman and Scott Ingram: The gortex suture in periareolar closure: A modified closure technique aesthetic Plast. Surg., 0696-8, 2016.

25- Donn M. Hickman: Application of Goes double-skin periareolar mastopexy with and without implants a 14years experience Journal of Plastic and Reconstructive Surgery, 64: 164-73, 2011.

26- Gryskiewicz J.M. and Agnieszka S. Hatfield, M.D.: Zigzag wavy-line periareolar incision, Vol., 110, 7, 2001. 